\title{
CORRIGENDUM: Genome variation and evolution of the malaria parasite Plasmodium falciparum
}

Daniel C Jeffares, Arnab Pain, Andrew Berry, Anthony V Cox, James Stalker, Catherine E Ingle, Alan Thomas, Michael A Quail, Kyle Siebenthall, Anne-Catrin Uhlemann, Sue Kyes, Sanjeev Krishna, Chris Newbold, Emmanouil T Dermitzakis \& Matthew Berriman Nat. Genet. 39, 120-125 (2007); published online 10 December 2006; corrected after print 8 February 2007

In the original version of this paper, the authors failed to acknowledge that sequencing of the P. falciparum IT laboratory isolate was funded by a European Union 6th Framework Program grant to the BioMalPar Consortium (contract number LSHP-LT-2004-503578). This error has been corrected in the PDF version of the article. 